\title{
Swiss World Atlas in Class Room - How to teach with maps
}

\author{
Wenke Zimmermann ${ }^{\mathrm{a}}{ }^{*}$, Lorenz Hurni ${ }^{\mathrm{b}}$ \\ ${ }^{a}$ ETH Zurich, Institute of Cartography and Geoinformation, Switzerland, w_zimmermann@ethz.ch \\ ${ }^{b}$ ETH Zurich, Institute of Cartography and Geoinformation, Switzerland, Ihurni@ethz.ch \\ * Corresponding author
}

Keywords: School Atlas, Education, Continuous Learning, Map Theory, Map Work, Map Competence

\begin{abstract}
:
The latest edition of the SWISS WORLD ATLAS, published in 2017, is coordinated with the didactic structure of the recently introduced geography curriculum on secondary school level in Switzerland. It represents an interdisciplinary tool for teaching and learning in geography lessons.

During the preparation and distribution phase of the new atlas, the number of enquiries about the conceptional and technical map production as well as didactical background information have increased. As the editorial office understand itself as a center of competence in maps, the idea of workshops about didactical map training developed. The purpose is to raise awareness what lies behind educational map work and map competence. As well as knowing the possible use cases of the SWISS WORLD ATLAS in class room, concrete effects of proper competence-orientated map work should be communicated.

In this contribution we present three didactic and teaching-orientated workshops. Each workshop addresses a different core topic and is scheduled for approx. 1 to 2 hours. The workshops can be shortened or extended depending on the scope of the event and it is possible to run them one after the other and link specific issues. Target groups are geography teachers and students.

One of those workshops deals with «map competences» in detail. The participants learn how to promote and practice the three sub-competences map reading, map understanding and map reflection. The focus lies on methodology teaching. As the proper handling of maps can be crucial in real life, further skills and proficiency need to be encouraged and trained.

Another workshop presents the synergy of the «printed edition and its online world». On the SWISS WORLD ATLAS website can be found a variety of supplementary material for each map (e.g. map comments, interactive tools). Participants discover how to integrate them in their teaching arrangement and add value to their classes in the form of media diversity and media literacy. Furthermore reference is made to a recently published teaching aid on lower secondary school level. This underlines even more the importance of combining innovative task culture and competence orientated learning in geography lessons.
\end{abstract}

The «atelier»-workshop is especially designed for students, who learn the conceptual background and successful workflow of an atlas product. With the help of typical tools and materials along the cartographic production line, the individual tasks from planning through design of maps, to the assembly of the atlas are experienced firsthand.

Up until now all workshops have been carried out several times, with minor changes due to event context or audience. All workshops support the comprehension of maps and the ability to accomplish competence-orientated knowledge, therefore the overall feedback is positive. Students get encouraged in their spatial orientation and cognition and are able to understand the basics of data preparation, map production and design and value the complexity of an atlas project. Whereas teachers can take home their elaborated documents and use the SWISS WORLD ATLAS immediately as a valuable tool in their practical map lessons. 